\title{
On Complete Cone Metric Space and Fixed Point Theorem
}

\author{
J. G. Mehta ${ }^{1 *}$ and M. L. Joshi ${ }^{2}$ \\ ${ }^{1}$ Harish Chandra Research Institute, Allahabad, India \\ ${ }^{2}$ Department of Mathematics, M. \& N. Virani Science College, Rajkot, India \\ Received 19 November 2010, accepted in final revised form 3 April 2011
}

\begin{abstract}
We prove coincidence and common fixed point theorems of four self mappings satisfying a generalized contractive type condition in complete cone metric spaces. Our results generalize some well-known recent results.

Keywords: Common fixed point; Complete cone metric space; Weakly compatible maps.

(c) 2011 JSR Publications. ISSN: 2070-0237 (Print); 2070-0245 (Online). All rights reserved.

doi:10.3329/jsr.v3i2.6475 J. Sci. Res. 3 (2), 303-309 (2011)
\end{abstract}

\section{Introduction}

The concept of cone metric space was introduced by Long-Guang and Xian [1], where the set of real numbers is replaced by an ordered Banach space. They introduced the basic definitions and discuss some properties of convergence of sequences in cone metric spaces. They also obtained various fixed point theorems for contractive single - valued maps in such spaces. Subsequently, some other mathematicians have generalized the results of Long-Guang and Zhang [1]. Afterwards, Rezapour and Hamlbarani [4] studied fixed point theorems of contractive type mappings by omitting the assumption of normality in cone metric spaces. Also many authors proved some fixed point theorems for contractive type mappings in cone metric [2, 5-7] spaces.

Wardowski [8] introduced the concept of multi valued contractions in cone metric spaces and, using the notion of normal cones, obtained fixed point theorems for such mappings. In 2008, Abbas and Jungck [2] proved some common fixed point theorems for weakly compatible mappings in the setting of a cone metric space.

The main purpose of this paper is to present fixed point results for contractive mappings in complete cone metric spaces.

\section{Preliminaries}

We recall the definitions of cone metric space, the notion of convergence and other results that will be needed in the sequel.

\footnotetext{
*Corresponding author: jgm_hri@yahoo.in
} 
Definition 2.1 Let $E$ be a real Banach Space and $P \subset E$. Then the set $P$ is called a cone if and only if

(i) $P$ is closed, non-empty and $P \neq\{\theta\}$;

(ii) $a, b \in R, a, b \geq 0, x, y \in P \Rightarrow a x+b y \in P$;

(iii) $P \cap(-P)=\{\theta\}$.

For a given cone $P \subset E$, we define a partial ordering $\leq$ with respect to $\mathrm{P}$ by $x \leq y$ if and only if $y-x \in P$. We shall write $x<y$ if $x \leq y$ and $x \neq y$, and $x \square y$ if $y-x \in \operatorname{Int}(P)$, where $\operatorname{Int}(P)$ denotes the interior of the set $P$.

Definition 2.2 [1] Let $E$ be a Banach Space and $P \subset E$ a cone. The cone $P$ is called normal if there is a number $\mathrm{k}>0$ such that for all $x, y \in E$,

$0 \leq x \leq y \Rightarrow\|x\| \leq k\|y\|$.

The least positive number $\mathrm{k}$ satisfying the above inequality is called the normal constant of $P$.

In the following we always suppose that $E$ is a Banach space, $P$ is a cone in $E$ with is partial ordering with respect to $P$.

Lemma 2.1 [1] If $P$ is a cone, $x \in P, \alpha \in R, 0 \leq \alpha<1$, and $x \leq \alpha x$, then $x=0$..

Proof. If $x \leq \alpha x$, then $\alpha x-x=(\alpha-1) x \in P$. Since $x \in P$ and $0 \leq \alpha<1$, we have, from Definition 1.1 (ii), (1- $\alpha) x \in P$.

It follows from Definition 1.1 (iii) that $x=0$.

In the following definition, we suppose that $E$ is a real Banach space, $P$ a cone in $E$ with $\operatorname{Int}(P) \neq \phi$ and that $\leq$ is the partial ordering with respect to $P$.

Definition 2.3 Let $X$ be a non-empty set. Suppose that the mapping $d: X \times X \rightarrow E$ satisfies following conditions:

(a) $0 \leq d(x, y)$ for all $x, y \in X$ and $d(x, y)=0$ if and only if $x=y$;

(b) $d(x, y)=d(y, x)$ for all $x, y \in X$;

(c) $d(x, y) \leq d(x, z)+d(y, z)$ for all $x, y, z \in X$.

Then $d$ is called a cone metric on $X$ and $(X, d)$ is called a cone metric space.

Notice that the notion of cone metric space is more general than the corresponding metric space followed by an example:

Example 2.1 Let $E=R^{2}, P=\{(x, y) \in E: x, y \geq 0\} \subset R^{2}, X=R$ and $d: X \times X \rightarrow E$ such that $\mathrm{d}(\mathrm{x}, \mathrm{y})=(|\mathrm{x}-\mathrm{y}|, \alpha|\mathrm{x}-\mathrm{y}|)$, where $\alpha \geq 0$ is a constant. Then $(\mathrm{X}, d)$ is a cone metric space.

Example 2.2 $E=R^{2}, P=\{(x, y) \in E: x, y \geq 0\} \subset R^{2}, X=R^{2}$ and $d: X \times X \rightarrow E$ such that

$$
\begin{aligned}
\mathrm{d}(\mathrm{x}, \mathrm{y}) & =\mathrm{d}\left(\left(\mathrm{x}_{1}, \mathrm{x}_{2}\right),\left(\mathrm{y}_{1}, \mathrm{y}_{2}\right)\right) \\
& =\left(\left|\mathrm{x}_{1}-\mathrm{y}_{1}\right|+\left|\mathrm{x}_{2}-\mathrm{y}_{2}\right|, \alpha \max \left\{\left|\mathrm{x}_{1}-\mathrm{y}_{1}\right|,\left|\mathrm{x}_{2}-\mathrm{y}_{2}\right|\right\}\right)
\end{aligned}
$$


where $\alpha \geq 0$ is a constant. Then $(X, d)$ is a cone metric space.

Definition 2.4 [1] Let $(X, d)$ be a cone metric space. Let $\left\{x_{n}\right\}$ be a sequence in $X$ and $x \in X$.

(i) $\left\{x_{n}\right\}$ converges to $x$ if for every $c \in E$ with $0 \square c$, there is an $n_{0} \in N$ such that for all $n \geq n_{0}, d\left(x_{n}, x\right) \square$ c.

We denote this by $\lim _{n \rightarrow \infty} x_{n} \rightarrow x$ or $x_{n} \rightarrow x(n \rightarrow \infty)$.

(ii) If for any $c \in E$ with $0 \square c$, there is an $n_{0} \in N$ such that

for all $n, m \geq n_{0} ; d\left(x_{n}, x_{m}\right) \square c$, then $\left\{x_{n}\right\}$ is called a Cauchy sequence in $X$

(iii) $(X, d)$ is called a complete cone metric space, if every Cauchy sequence in $X$ is convergent in $X$.

The proof of the following lemmas is very simple and need not to be discussed.

Lemma 2.2 [1] Let $(X, d)$ be a cone metric space. If $\left\{x_{n}\right\}$ is a convergent sequence in $X$, then the limit of $\left\{x_{n}\right\}$ is unique.

Lemma 2.3 Let $(X, d)$ be a cone metric space, $\left\{x_{n}\right\}$ a sequence in $X$. If $\left\{x_{n}\right\}$ converges to $x$ and $\left\{x_{n_{k}}\right\}$ is any subsequence of $\left\{x_{n}\right\}$, then $\left\{x_{n_{k}}\right\}$ converges to $x$.

Definition 2.5 Let $f$ and $g$ be two self-maps on a set $X$. Maps $f$ and $g$ are said to be commuting if $f g x=g f x$ for all $x \in X$.

Definition 2.6 Let $f$ and $g$ be self-maps on a set $X$. If $f x=g x$, for some $x$ in $X$ then $x$ is called coincidence point of $f$ and $g$.

Definition 2.7 [3] Let $f$ and $g$ be two self-maps defined on a set $X$. then $f$ and g are said to be weakly compatible if they commute at coincidence points. that is, if $f u=g u$ for some $u \in X$, then $f g u=g f u$.

Proposition 2.1 [2] Let $f$ and $g$ be weakly compatible self mappings of a set $X$. If $f$ and $g$ have a unique point of coincidence, that is, $w=f x=g x$, then $w$ is the unique common fixed point of $f$ and $g$.

\section{Main Result}

In this section we prove a common fixed point theorem for two pairs of weakly compatible mappings in complete cone metric spaces.

Theorem 3.1 Let $(X, d)$ be a complete cone metric space and $P$ be a normal cone with normal constant $k$. Suppose that the mappings $f, g, S$ and T are four self-maps of $X$ satisfying the following conditions:

(i) $T(X) \subseteq f(X)$ and $S(X) \subseteq g(X)$; 
(ii) $d(S x, T y) \leq a_{1} d(f x, S x)+a_{2} d(g y, S x)+a_{3} d(f x, T y)+a_{4} d(g y, T y)+a_{5}$ $d(f x, g y)$;

(iii) The pairs $(f, S)$ and $(g, T)$ are weakly compatible.

for all $x, y \in X$, where $a_{1}, a_{2}, a_{3}, a_{4}, a_{5} \geq 0$ and $a_{1}+a_{2}+2 a_{3}+2 a_{4}+a_{5}<1$.

Then $f, g, S$ and $T$ have a unique common fixed point.

Proof: Suppose $x_{0}$ is an arbitrary point of $X$ and define the sequence $\left\{y_{n}\right\}$ in $X$ such that,

$$
\begin{aligned}
& y_{2 n}=S x_{2 n}=g x_{2 n+1} \\
& y_{2 n+1}=T x_{2 n+1}=f x_{2 n+2}
\end{aligned}
$$

By (ii), we have,

$$
\begin{aligned}
& d\left(y_{2 n}, y_{2 n+1}\right)=d\left(S x_{2 n}, T x_{2 n+1}\right) \\
& \leq a_{1} d\left(f x_{2 n}, S x_{2 n}\right)+a_{2} d\left(g x_{2 n+1}, S x_{2 n}\right)+a_{3} d\left(f x_{2 n}, T x_{2 n+1}\right)+a_{4} d\left(g x_{2 n+1}, T x_{2 n+1}\right) \\
& \quad+a_{5} d\left(f x_{2 n}, g x_{2 n+1}\right) \\
& =a_{1} d\left(y_{2 n-1}, y_{2 n}\right)+a_{2} d\left(y_{2 n}, y_{2 n}\right)+a_{3} d\left(y_{2 n-1}, y_{2 n+1}\right)+a_{4} d\left(y_{2 n}, y_{2 n+1}\right)+ \\
& \quad a_{5} d\left(y_{2 n-1}, y_{2 n}\right) \\
& \leq a_{1} d\left(y_{2 n-1}, y_{2 n}\right)+0+a_{3}\left[d\left(y_{2 n-1}, y_{2 n}\right)+d\left(y_{2 n}, y_{2 n+1}\right)\right]+a_{4} d\left(y_{2 n}, y_{2 n+1}\right) \\
& \quad+a_{5} d\left(y_{2 n-1}, y_{2 n}\right) \\
& =\left(a_{1}+a_{3}+a_{5}\right) d\left(y_{2 n-1}, y_{2 n}\right)+\left(a_{3}+a_{4}\right) d\left(y_{2 n}, y_{2 n+1}\right)
\end{aligned}
$$

which implies that

$$
d\left(y_{2 n}, y_{2 n+1}\right) \leq h \quad d\left(y_{2 n-1}, y_{2 n}\right) \text { where } h=\frac{a_{1}+a_{3}+a_{5}}{1-\left(a_{3}+a_{4}\right)}<1 \text {. }
$$

Similarly it can be shown that

$$
d\left(y_{2 n+1}, y_{2 n+2}\right) \leq h d\left(y_{2 n}, y_{2 n+1}\right)
$$

Therefore; for all $n$,

$$
d\left(y_{n+1}, y_{n+2}\right) \leq h d\left(y_{n}, y_{n+1}\right) \leq \ldots \leq h^{n+1} d\left(y_{0}, y_{1}\right)
$$

Now for any $m>n$;

$$
\begin{aligned}
d\left(y_{n}, y_{m}\right) & \leq d\left(y_{n}, y_{n+1}\right)+d\left(y_{n+1}, y_{n+2}\right)+\ldots .+d\left(y_{m-1}, y_{m}\right) \\
& \leq\left(h^{n}+h^{n+1}+\ldots+h^{m-1}\right) d\left(y_{0}, y_{1}\right)
\end{aligned}
$$

By definition of normal cone, we have

$$
\left\|d\left(y_{n}, y_{m}\right)\right\| \leq \frac{\mathrm{h}^{\mathrm{n}}}{(1-\mathrm{h})} \mathrm{k}\left\|d\left(y_{1}, y_{0}\right)\right\|
$$

which implies that $d\left(y_{n}, y_{m}\right) \rightarrow 0$ as $n, m \rightarrow \infty$.

Hence $\left\{y_{n}\right\}$ is a Cauchy sequence in $X$.

Since $X$ is complete, there exists a point $z$ in $X$ such that $\lim _{n \rightarrow \infty} y_{n}=z$. 
Thus $\lim _{n \rightarrow \infty} S x_{2 n}=\lim _{n \rightarrow \infty} g x_{2 n+1}=z \quad$ and $\quad \lim _{n \rightarrow \infty} T x_{2 n+1}=\lim _{n \rightarrow \infty} f x_{2 n+2}=z$.

i.e. $\quad \lim _{n \rightarrow \infty} S x_{2 n}=\lim _{n \rightarrow \infty} g x_{2 n+1}=\lim _{n \rightarrow \infty} T x_{2 n+1}=\lim _{n \rightarrow \infty} f x_{2 n+2}=z$

Since $T(X) \subseteq f(X)$, there exists a point $u \in X$ such that $z=f u$.

Then, by (ii), we have

$$
\begin{aligned}
d(S u, z) \leq & \mathrm{d}\left(\mathrm{Su}, \mathrm{Tx}_{2 \mathrm{n}-1}\right)+\mathrm{d}\left(\mathrm{Tx}_{2 \mathrm{n}-1}, \mathrm{z}\right) \\
\leq & a_{1} \mathrm{~d}(\mathrm{fu}, \mathrm{Su})+a_{2} \mathrm{~d}\left(\mathrm{gx}_{2 \mathrm{n}-1}, \mathrm{Su}\right)+a_{3} \mathrm{~d}\left(\mathrm{fu}_{,} \mathrm{Tx}_{2 \mathrm{n}-1}\right)+a_{4} \mathrm{~d}\left(\mathrm{gx}_{2 \mathrm{n}-1}, \mathrm{Tx}_{2 \mathrm{n}-1}\right) \\
& \quad+a_{5} \mathrm{~d}\left(\mathrm{fu}, \mathrm{gx}_{2 \mathrm{n}-1}\right)+\mathrm{d}\left(\mathrm{Tx}_{2 \mathrm{n}-1}, \mathrm{z}\right)
\end{aligned}
$$

By definition of normal cone, we have

$$
\begin{aligned}
\|\mathrm{d}(\mathrm{Su}, \mathrm{z})\| \leq & a_{1} \mathrm{k}\|\mathrm{d}(\mathrm{fu}, \mathrm{Su})\|+a_{2} \mathrm{k}\left\|\mathrm{d}\left(\mathrm{gx}_{2 \mathrm{n}-1}, \mathrm{Su}\right)\right\|+a_{3} \mathrm{k}\left\|\mathrm{d}\left(\mathrm{fu} \mathrm{Tx}_{2 \mathrm{n}-1}\right)\right\| \\
& +a_{4} \mathrm{k}\left\|\mathrm{d}\left(\mathrm{gx}_{2 \mathrm{n}-1}, \mathrm{Tx}_{2 \mathrm{n}-1}\right)\right\|+a_{5} \mathrm{k}\left\|\mathrm{d}\left(\mathrm{fu} \mathrm{gx}_{2 \mathrm{n}-1}\right)\right\|+\mathrm{k}\left\|\mathrm{d}\left(\mathrm{Tx}_{2 \mathrm{n}-1}, \mathrm{z}\right)\right\|
\end{aligned}
$$

Taking the limit as $n \rightarrow \infty$ gives,

$$
\begin{aligned}
\mathrm{d}(\mathrm{Su}, \mathrm{z}) & \leq a_{1} \mathrm{~d}(z, \mathrm{Su})+a_{2} \mathrm{~d}(z, \mathrm{Su})+a_{3} \mathrm{~d}(z, \mathrm{z})+a_{4} \mathrm{~d}(z, \mathrm{z})+a_{5} \mathrm{~d}(z, \mathrm{z})+\mathrm{d}(z, \mathrm{z}) \\
& \leq\left(a_{1}+a_{2}\right) \mathrm{d}(z, \mathrm{Su})
\end{aligned}
$$

which is a contradiction since $a_{1}, a_{2}, a_{3}, a_{4}, a_{5} \geq 0$ and $a_{1}+a_{2}+2 a_{3}+2 a_{4}+a_{5}<1$

Therefore $S u=f u=z$.

Since $S(X) \subseteq g(X)$, there exists a point $v \in X$ such that $z=g v$.

Then, by (ii), we have

$$
\begin{aligned}
d(z, T v) & =d(S u, T v) \\
& \leq a_{1} d(f u, S u)+a_{2} d(g v, S u)+a_{3} d(f u, T v)+a_{4} d(g v, T v)+a_{5} d(f u, g v) \\
& =a_{1} \mathrm{~d}(z, \mathrm{z})+a_{2} \mathrm{~d}(z, \mathrm{z})+a_{3} d(z, T v) a_{4} d(z, T v)+a_{5} \mathrm{~d}(z, \mathrm{z}) \\
& =\left(a_{3}+a_{4}\right) d(z, T v)
\end{aligned}
$$

Thus $d(z, T v) \leq\left(a_{3}+a_{4}\right) d(z, T v)$

which is a contradiction since $a_{1}, a_{2}, a_{3}, a_{4}, a_{5} \geq 0$ and $a_{1}+a_{2}+2 a_{3}+2 a_{4}+a_{5}<1$

Therefore $T v=g v=z$.

Thus $f u=S u=T v=g v=z$.

Since $f$ and $S$ are weakly compatible maps, then $S f u=f S u$ i.e., $S z=f z$.

Now we show that $z$ is a fixed point of $S$.

If $S z \neq z$, then by (ii), we have

$d(S z, z)=d(S z, T v)$

$\leq a_{1} d(f z, S z)+a_{2} d(g v, S z)+a_{3} d(F z, T v)+a_{4} d(g v, T v)+a_{5} d(f z, g v)$

$=a_{1} d(S z, S z)+a_{2} d(z, S z)+a_{3} d(S z, z)+a_{4} d(z, z)+a_{5} d(S z, z)$

$=\left(a_{2}+a_{3}+a_{5}\right) d(S z, z)$ 
Thus $d(S z, z) \leq\left(a_{2}+a_{3}+a_{5}\right) d(S z, z)$, which is a contradiction since $a_{1}, a_{2}, a_{3}, a_{4}, a_{5} \geq 0$ and $a_{1}+a_{2}+2 a_{3}+2 a_{4}+a_{5}<1$

Therefore $S z=z$.

Hence $S z=f z=z$.

Similarly $g$ and $T$ are weakly compatible maps, we have $T z=g z$.

Now we show that $z$ is a fixed point of $T$.

If $T z \neq z$, then by (ii), we have

$d(z, T z)=d(S z, T z)$

$\leq a_{1} d(f z, S z)+a_{2} d(g z, S z)+a_{3} d(f z, T z)+a_{4} d(g z, T z)+a_{5} d(f z, g z)$

$=a_{1} \mathrm{~d}(z, \mathrm{z})+a_{2} d(T z, z)+a_{3} d(z, T z)+a_{4} d(T z, T z)+a_{5} d(z, T z)$

$=\left(a_{2}+a_{3}+a_{5}\right) d(T z, z)$

Thus $d(T z, z) \leq\left(a_{2}+a_{3}+a_{5}\right) d(T z, z)$

which is a contradiction since $a_{1}, a_{2}, a_{3}, a_{4}, a_{5} \geq 0$ and $a_{1}+a_{2}+2 a_{3}+2 a_{4}+a_{5}<1$

Therefore $T z=z$. Hence $T z=g z=z$.

Thus $S z=T z=f z=g z=z$, i.e. $z$ is a common fixed point of $f, g, S$ and $T$.

Uniqueness : For uniqueness of $z$ let if possible, we assume that $z$ and $w,(z \neq w)$ are common fixed points of $f, g, S$ and $T$,

then by (ii), we have

$$
\begin{aligned}
& \mathrm{d}(z, w)=d(S z, T w) \\
& \leq a_{1} d(f z, S z)+a_{2} d(g w, S z)+a_{3} d(f z, T w)+a_{4} d(g w, T w)+a_{5} d(f z, g w) \\
& =a_{1} \mathrm{~d}(z, z)+a_{2} \mathrm{~d}(w, z)+a_{3} \mathrm{~d}(z, w)+a_{4} \mathrm{~d}(w, w)+a_{5} \mathrm{~d}(z, w) \\
& =\left(a_{2}+a_{3}+a_{5}\right) \mathrm{d}(z, w)
\end{aligned}
$$

Thus $\mathrm{d}(z, w) \leq\left(a_{2}+a_{3}+a_{5}\right) \mathrm{d}(z, w)$

which is a contradiction since $a_{1}, a_{2}, a_{3}, a_{4}, a_{5} \geq 0$ and $a_{1}+a_{2}+2 a_{3}+2 a_{4}+a_{5}<1$

Therefore $z=w$.

Thus $z$ is the unique common fixed point of $f, g, S$ and $T$.

Hence the Theorem.

Corollary 3.2 Let $(X, d)$ be a complete cone metric space and $P$ be a normal cone with normal constant $k$. Suppose that the mappings, $f, s$ and $T$ are self-maps of $X$ satisfying the following conditions:

(i) $S(X) \cup T(X) \subseteq f(X)$;

(ii) $d(S x, T y) \leq$

$$
a_{1} d(f x, S x)+a_{2} d(f y, S x)+a_{3} d(f x, T y)+a_{4} d(f y, T y)+a_{5} d(f x, f y)
$$

(iii) The pairs $(f, S)$ and $(f, T)$ are weakly compatible.

for all $x, y \in X$, where $a_{1}, a_{2}, a_{3}, a_{4}, a_{5} \geq 0$ and $a_{1}+a_{2}+2 a_{3}+2 a_{4}+a_{5}<1$ 
Then $f, S$ and $T$ have a unique common fixed point.

Proof : By taking $f=g$ in theorem 3.1, we get the proof.

Corollary 3.3 Let $(X, d)$ be a complete cone metric space and $P$ be a normal cone with normal constant $k$. Suppose that the mappings $f$ and $S$ are self-maps of $X$ satisfying the following conditions:

(i) $S(X) \subseteq f(X)$;

(ii) $d(S x, S y) \leq$ $a_{1} d(f x, S x)+a_{2} d(f y, S x)+a_{3} d(f x, S y)+a_{4} d(f y, S y)+a_{5} d(f x, f y)$

(iii) The pair $(f, S)$ is weakly compatible, for all $x, y \in X$, where $a_{1}, a_{2}, a_{3}, a_{4}, a_{5} \geq 0$ and $a_{1}+a_{2}+2 a_{3}+2 a_{4}+a_{5}<1$ Then $f$ and $S$ have a unique common fixed point.

Proof: By taking $f=g$ and $S=T$ in theorem 3.1, we get the proof.

\section{Acknowledgement}

The authors are grateful to Professor J. N. Chauhan for his cooperation and valuable suggestions in the preparation of this paper.

\section{References}

1. H. Long-Guang and Z. Xian, J. Math. Anal. Appl. 332, 1468 (2007). doi:10.1016/j.jmaa.2005.03.087

2. M. Abbas and G. Jnngck, J. Math. Anal. Appl. 341, 416 (2008). doi:10.1016/j.jmaa.2007.09.070

3. G. Jungck and B. E. Rhoades, Indian J. Pure Appl. Math. 29 (3), 227 (1998).

4. S. Rezapour and R. Hamlbarani, J. Math. Anal. Appl. 345 (2), 719 (2008). doi:10.1016/j.jmaa.2008.04.049

5. D. Ilic and V. Rakocevic, J. Math. Anal. Appl. 341, 876 (2008). doi:10.1016/j.jmaa.2007.10.065

6. P. Raja, S. M. Vaezpour, Fixed Point Theory and Applications, Article ID 768294, 11 pages (2008).

7 I. Sahin and M. Telci, Hacet. J. Math. Stat. 38 (1), 59 (2009).

8 D. Wardowski, Nonlinear Analysis, Theory, Methods \& Applications 71, 512 (2009). 